\title{
Energy Expenditure Overestimation Bias in Elliptical Trainer Machine
}

\author{
KELSEY MCLAUGHLIN", BETHANY NOACK¹, JORGE Z GRANADOS1, RICK \\ SPERLING ${ }^{2}$, MARK ROLTSCH${ }^{3}$, and STEPHEN F CROUSE1
}

${ }^{1}$ Applied Exercise Physiology Laboratory; Department of Kinesiology; Texas A\&M University; College Station, TX 2Department of Psychology; St. Mary's University; San Antonio, TX ${ }^{3}$ Department of Exercise Science and Community Health; University of West Florida; Pensacola, FL

\section{Category: Masters \\ Advisor / Mentor: Crouse, Stephen(s-crouse@tamu.edu)}

ABSTRACT

Elliptical trainers are a common mode of aerobic exercise in recreationally active populations. Those with a weight loss goal might rely upon the energy expenditure (EE) estimation that many elliptical brands provide to keep track of calories (kcals) burned and make nutritional decisions. For this reason, it is important to evaluate the accuracy of the algorithms used by elliptical trainers to estimate EE. The purpose of this study was to compare EE estimates by a common brand of elliptical trainer to that measured using open circuit spirometry, at different combinations of resistance and pedal speed. Twenty subjects (10 male, 10 female; $34 \pm 12$ yr; $175.3 \pm 10.7 \mathrm{~cm} ; 77.1 \pm 14.1 \mathrm{~kg}$ ) consented to participate. Each completed three 15-min bouts of elliptical exercise on the same elliptical trainer, with at least $24 \mathrm{hr}$ between exercise bouts. Pedal rates were held constant throughout each bout at 50,60, or $70 \mathrm{RPM}$, and resistance was increased incrementally every $5 \mathrm{~min}$ from level 5 to 10 to 15 . The different cadences were completed in a randomized order between participants. Expired gases were collected continuously throughout the $15 \mathrm{~min}$. Heart rate, distance (mi), and EE from the elliptical readout were recorded every $1 \mathrm{~min}$. RPE was collected twice per resistance level. A two-tailed paired samples t-test was used to compare elliptical EE to measured EE. A linear regression model was used to evaluate the ability of the elliptical EE to predict measured EE. Significance for all statistical measures was held at an alpha level of 0.05 . The difference between EE estimates from the elliptical and measured VO2 was significant $(p<0.0001)$, with the elliptical machine overestimating EE during a 15 minute session by an average of 10.21 kcals. Measured EE in kcals as derived from open circuit spirometry was significantly predicted by elliptical EE according to the equation:

Measured EE $=0.95 *($ Elliptical EE $)-3.161$

In conclusion, the elliptical trainer used for this study demonstrated a bias to overestimate EE. This should be taken into account by health/fitness professionals using these estimations to program for clients. There may be some variation in the EE correction regression depending on elliptical model, and proper machine calibration should be ensured. 